\title{
THE ENVIRONMENTS OF ACTIVE GALACTIC NUCLEI WITHIN THE zCOSMOS DENSITY FIELD
}

\author{
J. D. Silverman ${ }^{1}$, K. KovaČ ${ }^{1}$, C. Knobel ${ }^{1}$, S. Lillly $^{1}$, M. Bolzonella ${ }^{2}$, F. Lamareille ${ }^{3}$, V. Mainieri ${ }^{4}$, M. Brusa ${ }^{5}$, \\ N. Cappellutit ${ }^{5}$, Y. Peng ${ }^{1}$, G. Hasinger ${ }^{5}$, G. Zamorani ${ }^{2}$, M. Scodeggio ${ }^{6}$, T. Contini ${ }^{3}$, C. M. Carollo ${ }^{1}$, K. JahnKe ${ }^{7}$, \\ J.-P. Kneib ${ }^{8}$, O. Le Fevre ${ }^{8}$, S. Bardelli ${ }^{2}$, A. Bongiorno ${ }^{5}$, H. Brunner ${ }^{5}$, K. Caputi ${ }^{1}$, F. Civano ${ }^{9}$, A. Comastri ${ }^{2}$, G. Coppa ${ }^{2}$, \\ O. Cucciati ${ }^{10}$, S. de la Torre ${ }^{8}$, L. De Ravel ${ }^{8}$, M. Elvis ${ }^{11}$, A. Finoguenov ${ }^{1}$, F. Fiore ${ }^{12}$, P. Franzetti ${ }^{6}$, B. Garilli ${ }^{6}$, R. Gilli $^{2}$, \\ R. Griffiths ${ }^{13}$, A. Iovino ${ }^{10}$, P. KAMPCZYK ${ }^{1}$, A. KoeKemoer ${ }^{19}$, J.-F. Le Borgne ${ }^{3}$, V. Le Brun ${ }^{8}$, C. Maier ${ }^{1}$, M. MignOli ${ }^{2}$, \\ R. Pello ${ }^{3}$, E. Perez Montero ${ }^{3}$, E. Ricciardelli ${ }^{14}$, M. Tanaka ${ }^{4}$, L. Tasca ${ }^{8}$, L. Tresse ${ }^{8}$, D. Vergani ${ }^{2}$, C. Vignali $^{15}$, \\ E. Zucca ${ }^{2}$, D. Bottini ${ }^{6}$, A. Cappi ${ }^{2}$, P. Cassata ${ }^{8}$, C. Marinoni ${ }^{16}$, H. J. McCracken ${ }^{17}$, P. Memeo ${ }^{6}$, B. Meneux ${ }^{5,9}$, P. Oesch $^{1}$, \\ C. Porciani ${ }^{1}$, and M. Salvato ${ }^{18}$ \\ Institute of Astronomy, ETH Zürich, CH-8093, Zürich, Switzerland \\ INAF Osservatorio Astronomico di Bologna, via Ranzani 1, I-40127, Bologna, Italy \\ Laboratoire d'Astrophysique de Toulouse-Tarbes, Université de Toulouse, Centre National de la Recherche Scientifique, 14 Avenue Edouard Belin, \\ F-31400 Toulouse, France \\ European Southern Observatory, Karl-Schwarzschild-Strasse 2, Garching, D-85748, Germany \\ Max-Planck-Institut für extraterrestrische Physik, D-84571 Garching, Germany \\ INAF, IASF Milano, Milan, Italy \\ Max-Planck-Institut für Astronomie, Königstuhl 17, D-69117 Heidelberg, Germany \\ Laboratoire d'Astrophysique de Marseille, Marseille, France \\ Universitats-Sternwarte, Scheinerstrasse 1, D-81679 Muenchen, Germany \\ INAF Osservatorio Astronomico di Brera, Milan, Italy \\ Harvard-Smithsonian Center for Astrophysics, 60 Garden Street, Cambridge, MA 02138, USA \\ INAF, Osservatorio di Roma, Monteporzio Catone (RM), Italy \\ Department of Physics, Carnegie Mellon University, 5000 Forbes Avenue, Pittsburgh, PA 15213, USA \\ Dipartimento di Astronomia, Universita di Padova, Padova, Italy \\ Dipartimento di Astronomia, Universitá di Bologna, via Ranzani 1, I-40127, Bologna, Italy \\ Centre de Physique Theorique, Marseille, Marseille, France \\ Institut d'Astrophysique de Paris, UMR 7095 CNRS, Université Pierre et Marie Curie, 98 bis Boulevard Arago, F-75014 Paris, France \\ California Institute of Technology, Pasadena, CA, USA \\ Space Telescope Science Institute, 3700 SanMartin Drive, Baltimore, MD 21218, USA \\ Received 2008 October 24; accepted 2009 January 2; published 2009 March 30
}

\begin{abstract}
The impact of environment on active galactic nucleus (AGN) activity up to $z \sim 1$ is assessed by utilizing a mass-selected sample of galaxies from the 10k catalog of the zCOSMOS spectroscopic redshift survey. We identify 147 AGN by their X-ray emission as detected by XMM-Newton from a parent sample of 7234 galaxies. We measure the fraction of galaxies with stellar mass $M_{*}>2.5 \times 10^{10} M_{\odot}$ that host an AGN as a function of local overdensity using the 5th, 10th, and 20th nearest neighbors that cover a range of physical scales ( $\sim 1-4 \mathrm{Mpc})$. Overall, we find that AGNs prefer to reside in environments equivalent to massive galaxies with substantial levels of star formation. Specifically, AGNs with host masses between 0.25 and $1 \times 10^{11} M_{\odot}$ span the full range of environments (i.e., field to group) exhibited by galaxies of the same mass and rest-frame color or specific star formation rate. Host galaxies having $M_{*}>10^{11} M_{\odot}$ clearly illustrate the association with star formation since they are predominantly bluer than the underlying galaxy population and exhibit a preference for lower-density regions analogous to Sloan Digital Sky Survey studies of narrow-line AGN. To probe the environment on smaller physical scales, we determine the fraction of galaxies $\left(M_{*}>2.5 \times 10^{10} M_{\odot}\right)$ hosting AGNs inside optically selected groups, and find no significant difference with field galaxies. We interpret our results as evidence that AGN activity requires a sufficient fuel supply; the probability of a massive galaxy to have retained some sufficient amount of gas, as evidence by its ongoing star formation, is higher in underdense regions where disruptive processes (i.e., galaxy harassment, tidal stripping) are lessened.
\end{abstract}

Key words: galaxies: active - quasars: general - X-rays: galaxies

Online-only material: color figures

\section{INTRODUCTION}

The local environment of galaxies harboring active galactic nuclei (AGNs) and QSOs has long been thought to play a potential role in triggering mass accretion onto supermassive black holes (SMBHs). With many of the properties of galaxies (e.g., morphology, color, star formation rate (SFR)) clearly dependent on environment (e.g., Kauffmann et al. 2004; Baldry et al. 2006; Cooper et al. 2006; Cucciati et al. 2009, in preparation) and the possibility of a common history of mass assembly for SMBHs and their host bulges (e.g., Granato et al. 2004; Bower et al. 2006; Hopkins et al. 2008; Somerville et al. 2008), we expect that AGNs may prefer to reside in specific environments most nurturing for their growth. Identifying environmental factors might allow us to determine the physical mechanism(s) responsible for driving accretion such as major mergers of galaxies that has been demonstrated through numerical simulations to be able to remove angular momentum from rotationally supported gas thus transferring mass to the nuclear regions (e.g. Barnes \& Hernquist 1996; Mihos \& Hernquist 1996). For 
example, there is evidence that AGNs reside in dark matter halos with masses $M_{\text {halo }} \sim 10^{12-13} M_{\odot}$ (Porciani et al. 2004; Hopkins et al. 2007; Mandelbaum et al. 2008; Pasquali et al. 2009; Bonoli et al. 2008), a mass regime comparable to the groupscale environments thought to be fertile ground for galaxies to coalesce. As well, high-density regions such as massive clusters of galaxies are expected to be inhospital environments for AGN (Dressler et al. 1985) given the strong empirical association between AGN activity and concurrent star formation (e.g., Kauffmann et al. 2003; Jahnke et al. 2004; Silverman et al. 2009) although counterevidence exists for the radio-loud population (Hill \& Lilly 1991; Best et al. 2007).

Environmental studies to date have presented seemingly disparate results most likely due to varying selection methods and physical scales used to characterize environment. Using a large sample of narrow-line AGN from the Sloan Digital Sky Survey (SDSS), Miller et al. (2003) find that there is no environmental dependence on AGN activity using a magnitude-limited sample of galaxies and meaning environment on scales of around a few megaparsecs. Upon further investigation, Kauffmann et al. (2004) find that an environmental dependence, similar to star formation, emerges when implementing a selection based on stellar mass and considering the luminosity of the AGN. Supportive of this scenario, Coil et al. (2007) find that quasars in the DEEP2 survey fields have environments similar to blue galaxies. On the other hand, luminous quasars from the SDSS have an overabundance of galaxies within their vicinity on smaller scales (less than 0.1 Mpc; Serber et al. 2006) that is in agreement with clustering analysis based on quasar pairs (Hennawi et al. 2006) but may still have significant biases in the employed methods (Padmanabhan et al. 2008). Furthermore, the enhancement in star formation attributed to galaxy mergers on scales of $0.01-0.1 \mathrm{kpc}$ is not reflected in AGN accretion (Li et al. 2008) for which the authors conclude may be due to varying timescales with star formation preceding AGN activity (e.g., Schawinski et al. 2007) occurring within a more relaxed host galaxy.

$\mathrm{X}$-ray-selected surveys with both Chandra and XMM-Newton now enable the study of the environment of AGNs including the obscured population at higher redshifts $(z \gtrsim 0.3)$ where optical selection of narrow-line AGN is difficult. Grogin et al. (2005) first looked into the environments of X-ray-selected sources detected in the Chandra Deep Fields and found that the nearneighbor counts were identical to the galaxies without AGN. This led the authors to conclude that mergers were not the physical mechanism triggering mass accretion especially since the AGN host galaxies were no more asymmetric than the average galaxy of equivalent luminosity (see Gabor et al. 2009, for a morphological study of AGN hosts in COSMOS). While Georgakakis et al. (2007) claim based on a large spectroscopic sample of galaxies from DEEP2 that X-ray-selected AGNs at $z \sim 1$ prefer higher-density environments, their results are consistent with those of galaxies having similar host properties (i.e., absolute magnitude, rest-frame color). From a complementary perspective, Martini et al. (2007) have measured the AGN content of galaxy clusters and found no significant difference with the fraction of field galaxies hosting AGN. Recently, Gilli et al. (2009) found clustering lengths of AGN in the COSMOS field comparable to massive galaxies and concluded that the clustering signal is reflective of SMBHs preferring to reside in galaxies with $M_{*}>3 \times 10^{10} M_{\odot}$. To date, an environmental analysis of X-ray-selected AGN based on both a large spectroscopic survey of galaxies and careful consideration of selection to disentangle the degeneracy between mass and environment seen in galaxy studies (e.g., Baldry et al. 2006; van der Wel 2008; Cucciati et al. 2009, in preparation) has not yet been attempted.

Here, we utilize the rich multiwavelength observations of the COSMOS field (Scoville et al. 2007) to determine the role of environment in triggering AGN at $0.1<z<1.0$. The COSMOS survey is roughly a $2 \mathrm{deg}^{2}$ region of the sky selected to have full coverage with all major observatories both from the ground (i.e., Subaru, Very Large Telescope (VLT)) and space (e.g., Hubble Space Telescope (HST), Spitzer, XMM-Newton). The zCOSMOS survey (Lilly et al. 2007; S. J. Lilly et al. 2009 , in preparation) targets objects for optical spectroscopy with the VLT in two separate observing programs. A "bright" sample $\left(i_{\mathrm{ACS}}<22.5\right)$ is observed with a red grism to provide a wavelength coverage of 5500-9500 $\AA$ ideal to identify galaxies $\left(L_{*}\right)$ up to $z \sim 1.2$. A deeper program, not utilized in the present study, targets faint galaxies $(B<25)$, selected to be in the redshift range $1.5 \lesssim z \lesssim 2.5$ using a blue grism for an effective wavelength coverage of $3600<\lambda<6700 \AA$. For the present study, we select galaxies based on reliable spectroscopic redshifts from the zCOSMOS "bright" program and their stellar mass estimates based on broadband photometry. Those that host AGNs are identified by their X-ray emission as detected by XMM-Newton (Hasinger et al. 2007; Cappelluti et al. $2007,2009)$. We use the nearest-neighbor approach to determine the projected local galaxy density using the three-dimensional galaxy distribution as fully described in Kovač et al. (2009). In addition, the zCOSMOS optically selected group catalog (Knobel et al. 2009) offers a complementary perspective on the role of environment. The low optical luminosity and obscured AGN further enable us to determine if the host galaxies of AGN exhibit a trend similar to the color-density or SFR-density relations found for nonactive galaxies. Finally, we refer the reader to Silverman et al. (2009) that presents the properties (i.e., stellar mass, SFR, rest-frame color) of the hosts of X-rayselected AGN in the COSMOS field.

Throughout this work, we assume $H_{0}=70 \mathrm{~km} \mathrm{~s}^{-1} \mathrm{Mpc}^{-1}$, $\Omega_{\Lambda}=0.75, \Omega_{\mathrm{M}}=0.25$ and use AB magnitudes.

\section{DATA AND DERIVED PROPERTIES}

\subsection{Parent Galaxy Sample}

In order to compare the environments of AGN hosts with those of normal galaxies, we use for the latter the well-defined "parent sample" of galaxies up to $z \sim 1$ from the zCOSMOS 10k spectroscopic "bright" catalog, as done in Silverman et al. (2009). Specifically, we select 7234 galaxies with an apparent magnitude $i_{\mathrm{ACS}}<22.5$, and a redshift $0.1<z<1.0$ having a spectroscopic redshift quality flag greater than or equal to 2.0 (see Lilly et al. 2007) that amounts to a confidence of $~ 99 \%$ for the overall sample. The spatial sampling as shown in Figure 1 is fairly uniform across the central $1 \mathrm{deg}^{2}$ while the completed zCOSMOS 20k catalog will fill in the gaps mainly located along the perimeter of the COSMOS field. Further details on the spatial sampling and quality assurance can be found in Lilly et al. (2007) and S. J. Lilly et al. (2009, in preparation) as well as a complete description of target assignments, data acquisition, and the subsequent reduction procedure based on the VIMOS Interactive Pipeline and Graphical Interface package (VIPGI; Scodeggio et al. 2005).

Stellar masses, including rest-frame absolute magnitudes $\left(M_{U}, M_{V}\right)$ in the AB system, are derived from fitting stellar population synthesis models from the library of Bruzual \& 
Table 1

Sample Statistics-Density Field Analysis

\begin{tabular}{|c|c|c|c|c|c|}
\hline Sample & $\begin{array}{l}\text { Mass }^{\mathrm{a}} \\
\text { Range } \\
\end{array}$ & $\begin{array}{l}\text { Redshift } \\
\text { Range }\end{array}$ & No. of Galaxies & $\begin{array}{c}\text { No. of AGNs } \\
\left(L_{0.5-10 \mathrm{keV})}\right.\end{array}$ & Use \\
\hline All & $\ldots$ & $0.1<z<1.0$ & 7234 & $147(>42)$ & Total sample \\
\hline A & $>10.4$ & $0.1<z<1.0$ & 2457 & $63(42.48-43.7) ; 88(>42.48)$ & AGN fraction \\
\hline $\mathrm{B}$ & $10.4-11$ & $0.1<z<1.0$ & 1971 & $48(42.48-43.7)$ & AGN fraction \\
\hline $\mathrm{C}$ & $>11$ & $0.1<z<1.0$ & 486 & $14(42.48-43.7) ; 20(>42.48)$ & AGN fraction \\
\hline $\mathrm{D}$ & $>10.2$ & $0.1<z<0.8$ & 2482 & $77(42-43.7)$ & Color-density relation \\
\hline
\end{tabular}

Notes.

${ }^{\mathrm{a}}$ Units of $\log M_{\odot}$.

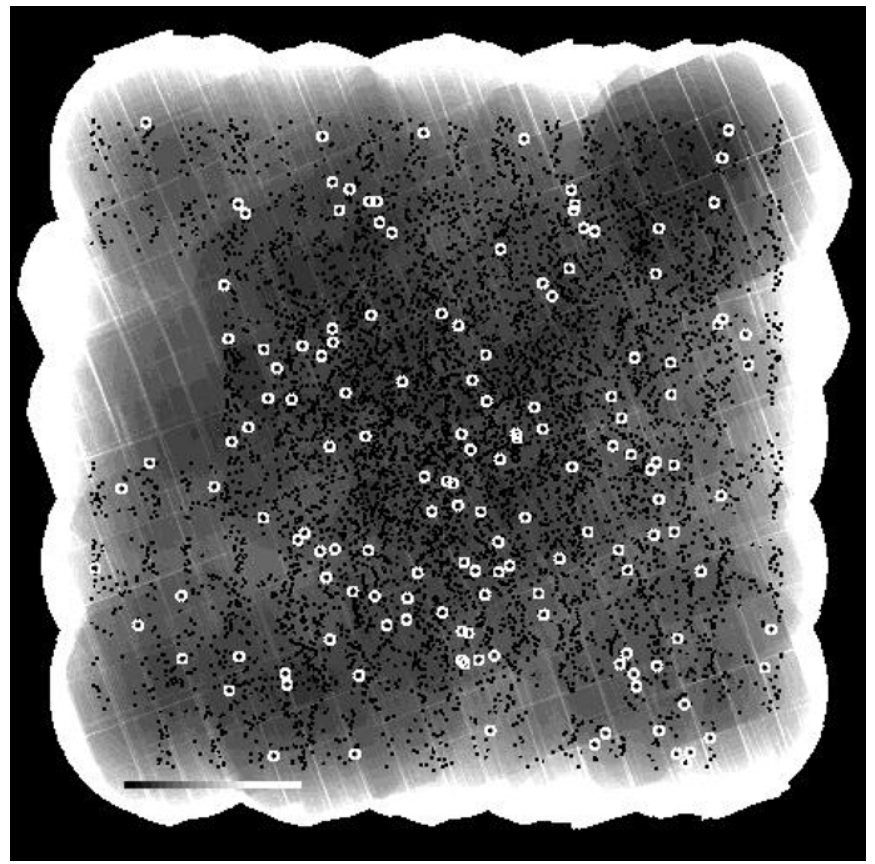

Figure 1. Spatial distribution of 7234 zCOSMOS galaxies (small black dots) with $i_{\mathrm{ACS}}<22.5$ and $0.1<z<1.0$. Those identified as AGN based on $X M M$-Newton detections $\left(147 ; L_{0.5}-10 \mathrm{keV}>10^{42} \mathrm{erg} \mathrm{s}^{-1}\right.$ ) are highlighted with a larger white circle. The underlying gray-scale image is the exposure map of the XMM-Newton mosaic (north is up and east is to the left). A scale bar ( $20^{\prime}$ in length) ranges from $1 \times 10^{-15}-1 \times 10^{-14} \mathrm{erg} \mathrm{cm}^{-2} \mathrm{~s}^{-1}$.

Charlot (2003) to both the broadband optical (CFHT: $u, i, K_{s}$; Subaru: $B, V, g, r, i, z$; Capak et al. 2007) and near-infrared (Spitzer/IRAC: $3.6 \mu, 4.5 \mu$; Sanders et al. 2007) photometry using a chi-square minimization for each galaxy. The measurement of stellar mass $\left(M_{*}\right)$ includes (1) the assumption of a Chabrier initial mass function ${ }^{20}$, (2) a star formation history with both a constant rate and an additional exponentially declining component covering a range of time scales $(0.1<\tau<30 \mathrm{Gyr})$, (3) extinction $\left(0<A_{V}<3\right)$ following Calzetti et al. (2000), and (4) solar metallicities. In Figure 2a, we show the distribution of stellar mass versus redshift for our sample. Further details on mass measurements of zCOSMOS galaxies can be found in M. Bolzonella et al. (2009, in preparation), Meneux et al. (2009), and L. Pozzetti (2009, in preparation).

To minimize any selection biases, we determine a minimum mass threshold that all galaxies must satisfy. The mass limit is set to ensure a fairly complete representation of both blue and red galaxies at all redshifts considered. Meneux et al. (2009)

\footnotetext{
${ }^{20}$ Masses are not corrected to those based on a Salpeter IMF as done in
} Silverman et al. (2009). estimate based on a series of mock catalogs from the millennium simulation that the zCOSMOS "Bright sample" is essentially complete for galaxies with $\log M_{*} \approx 10.6$ at $z=0.8$ while the completeness drops to $\sim 50 \%$ at $z=1$. We impose a slightly lower-mass limit of $\log M_{*}>10.4$ (units of $M_{\odot}$ ) to provide a fair representation of galaxies covering the full range of restframe color $U-V$ up to $z \sim 1$ and ensure an adequate sample that host AGN (see below). In Figure 2b, it is evident that this mass limit is essentially imposed by the red galaxy population due to the initial selection on apparent magnitude. Above this mass limit, we have a sample of 2457 galaxies, over the redshift range $0.1<z<1.0$ (Table 1 ).

Further spectroscopic measurements such as emission and absorption line strengths, and continuum indices are performed through an automated pipeline ("platefit_vimos"; Lamareille et al. 2008) similar to that performed with the SDSS (Tremonti et al. 2004). For the present study, we specifically use the [O II] 33727 emission-line luminosity, corrected for slit loss and an AGN contribution if present, to determine the mass-weighted (specific) SFR (sSFR) using the empirical relation given in Moustakas et al. (2006) thus allowing us to investigate the environmental dependence of star formation for galaxies hosting AGN. We refer the reader to Maier et al. (2009) and Silverman et al. (2009) for more details regarding spectral measurements of zCOSMOS galaxies and the removal of AGN emission based on the observed or inferred [O III] $\lambda 5007$ line flux (M. Bolzonella et al. 2009, in preparation; B. Meneux et al. 2009, in preparation).

\subsection{AGN Identification}

The $X M M$ observations of the COSMOS field (Hasinger et al. 2007) enable us to identify those galaxies from the aforementioned zCOSMOS parent sample of mass-selected galaxies that harbor AGN. Briefly, a zCOSMOS galaxy has associated $\mathrm{X}$-ray emission if a maximum likelihood routine (Brusa et al. 2007) provides a clear association with an X-ray detection in either the soft-band $\left(f_{0.5-2 \mathrm{keV}}>5 \times 10^{-16} \mathrm{erg} \mathrm{cm}^{-2} \mathrm{~s}^{-1}\right)$ or hard-band $\left(f_{2-10 \mathrm{keV}}>2 \times 10^{-15} \mathrm{erg} \mathrm{cm}^{-2} \mathrm{~s}^{-1}\right)$ catalogs (Cappelluti et al. 2009). Out of 7234 galaxies, we identify 153 as having significant $\mathrm{X}$-ray emission. We note that the frequency of X-ray sources with optical spectra is higher than would be seen in the general population because a subset of these sources were designated as "compulsory" during the design of VIMOS masks and therefore observed at about twice the sampling rate of the random targets. This "bias" does not depend on any other property of the galaxy. We account for this when necessary such as measuring the fraction of galaxies hosting AGN. We refer the reader to Brusa et al. (2007) and Silverman et al. (2009) for more explicit details 

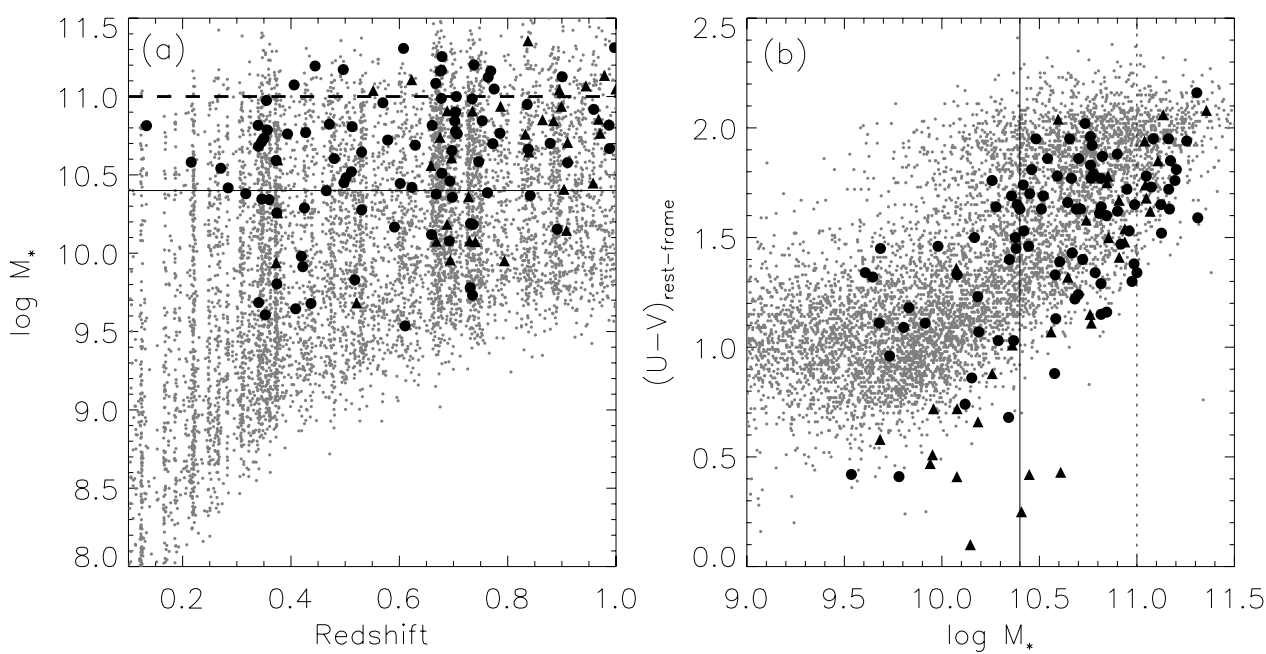

Figure 2. (a) Stellar mass vs. redshift for 7234 zCOSMOS galaxies $(0.1<z<1.0$, small gray circles). (b) Rest-frame color $U-V$ vs. stellar mass. The mass limits for two samples are highlighted in both panels: $\log M_{*}>10.4$ (solid line) and $\log M_{*}>11$ (dashed line). Galaxies hosting X-ray-selected AGN (147) are marked by a larger black symbol with respect to their X-ray luminosity (circles: $42<\log L_{0.5-10 \mathrm{keV}}<43.7$; triangles: $\log L_{0.5-10 \mathrm{keV}}>43.7$ ) in both panels.

regarding X-ray detection, optical source matching, and followup spectroscopy.

Our criteria for determining whether X-ray emission is due to an AGN is based on its pointlike spatial extent and whether the luminosity is above $10^{42} \mathrm{erg} \mathrm{s}^{-1}$ in either X-ray energy band. Most X-ray sources in our sample have luminosities above this threshold as evident in Figure 1 of Silverman et al. (2009) that shows the equivalent data set. Therefore, we attribute 147 of the 153 galaxies with X-ray emission to be the result of an AGN and mark their spatial distribution in Figure 1. Our X-ray-selected AGNs mainly have $42 \lesssim \log L_{X} \lesssim 44$ with a few being more luminous (i.e., QSOs). Since the majority of these AGNs are optically underluminous, we can investigate if the properties of their host galaxies (i.e., mass, rest-frame color, SFR) depend on environment without too much concern for AGN contamination especially for those with $\log L_{0.5-10 \mathrm{keV}}<43.7$. This upper limit is based on an empirical relation (Silverman et al. 2005) where optical emission, associated with X-ray-selected AGN at $\log v l_{v}<43.3$ at $E=2 \mathrm{keV}$ is primarily due to their host galaxy since there is a strong departure from the known $l_{\mathrm{opt}}-l_{\mathrm{X}}$ relation for more-luminous AGN. We convert this monochromatic luminosity to a value of $10^{43.7} \mathrm{erg} \mathrm{s}^{-1}$ in the broad band (0.5-10.0 keV) assuming a power-law spectrum with photon index $\Gamma=1.9$. The lack of broad emission lines for most of our samples (see Figure 1 of Silverman et al. 2009) within this restricted luminosity range also lends support that these objects are dominated in the optical by their host galaxy. Here, we further highlight in Figure 2 the galaxies that harbor $\mathrm{X}$-ray-selected AGN with respect to the stellar mass ${ }^{21}$ and restframe color. We note that the overdensity of AGNs at $z \sim 0.7$ (Figure 2(a)) is indicative of an underlying number density of galaxies and not an enhancement of AGN activity in large-scale structures (Silverman et al. 2008a) although a full assessment of such effects will be explored in a future study having improved statistics.

We remark that a significant sample of additional AGN has been spectroscopically identified through the Magellan/IMACS

\footnotetext{
21 It is worth mentioning, as done in Silverman et al. (2009), that there may be a potential problem that galaxies with even moderate-luminosity AGN may have inaccurate mass estimates; a bluer continuum will essentially reduce the stellar age and hence lower the mass measurements since the derived mass-to-light ratio depends strongly on the spectrum.
}

observing program (Trump et al. 2007) that can effectively improve upon the sample. For the current quantitative analysis, we choose to use only zCOSMOS identifications in order to maintain a high degree of uniformity and well-defined relation to a parent sample of galaxies. We will explore the feasibility of incorporating such samples in a future investigation.

\section{3. zCOSMOS Galaxy Density Field}

A major aim of the zCOSMOS survey is to reconstruct the three-dimensional density field using the "bright" sample to characterize the environment up to $z \sim 1$ and discern its impact on galaxy evolution. Here, we briefly outline the methodology and refer the reader to Kovač et al. (2009) for full details regarding the galaxy density estimates in the zCOSMOS field. The procedure is based on the algorithm "Zurich Adaptive Density Estimator" (ZADE) that utilizes spectroscopic (10k) and photometric redshifts (30k) for accurate distances to practically all galaxies with $i_{\mathrm{ACS}}<22.5$. For galaxies without spectroscopic redshifts, their photometric redshift likelihood functions are adjusted using galaxies in the spectroscopic catalog that are closely along the line of sight and within the effective aperture. This procedure effectively accounts for the incomplete sampling ( $\sim 30 \%)$ of the current $10 \mathrm{k}$ catalog thus improving density estimates throughout the zCOSMOS volume. We use density estimates based on a flux-limited catalog and adaptive apertures (based on nearest neighbors) in order to maintain a reasonable sample of AGNs over the full redshift range $(0.1<z<1.0)$ at the expense of having a redshift-dependent smoothing scale (see Figure 6 of Kovač et al. 2009). To minimize redshift-space distortions induced by the intrinsic velocity dispersion of groups and clusters, a projection of $\pm 1000 \mathrm{~km} \mathrm{~s}^{-1}$ in redshift space is implemented. Finally, we express the quantitative measure of the environment as an overdensity $\left(\delta\right.$; where $\left.1+\delta=\rho\langle\rho(z)\rangle^{-1}\right)$ at the position of each galaxy relative to the mean density $(\langle\rho(z)\rangle)$ at a given redshift. Error estimates are obtained by comparison with reconstructed overdensities from mock catalogs (Kitzbichler \& White 2007) extracted from the Millennium simulation (Springel et al. 2005) and tailored to match the current zCOSMOS sample. Typical errors on $\log (1+\delta)$ are between 0.1 and 0.15 over a wide range of overdensity (see Figure 4 of Kovač et al. 2009). 

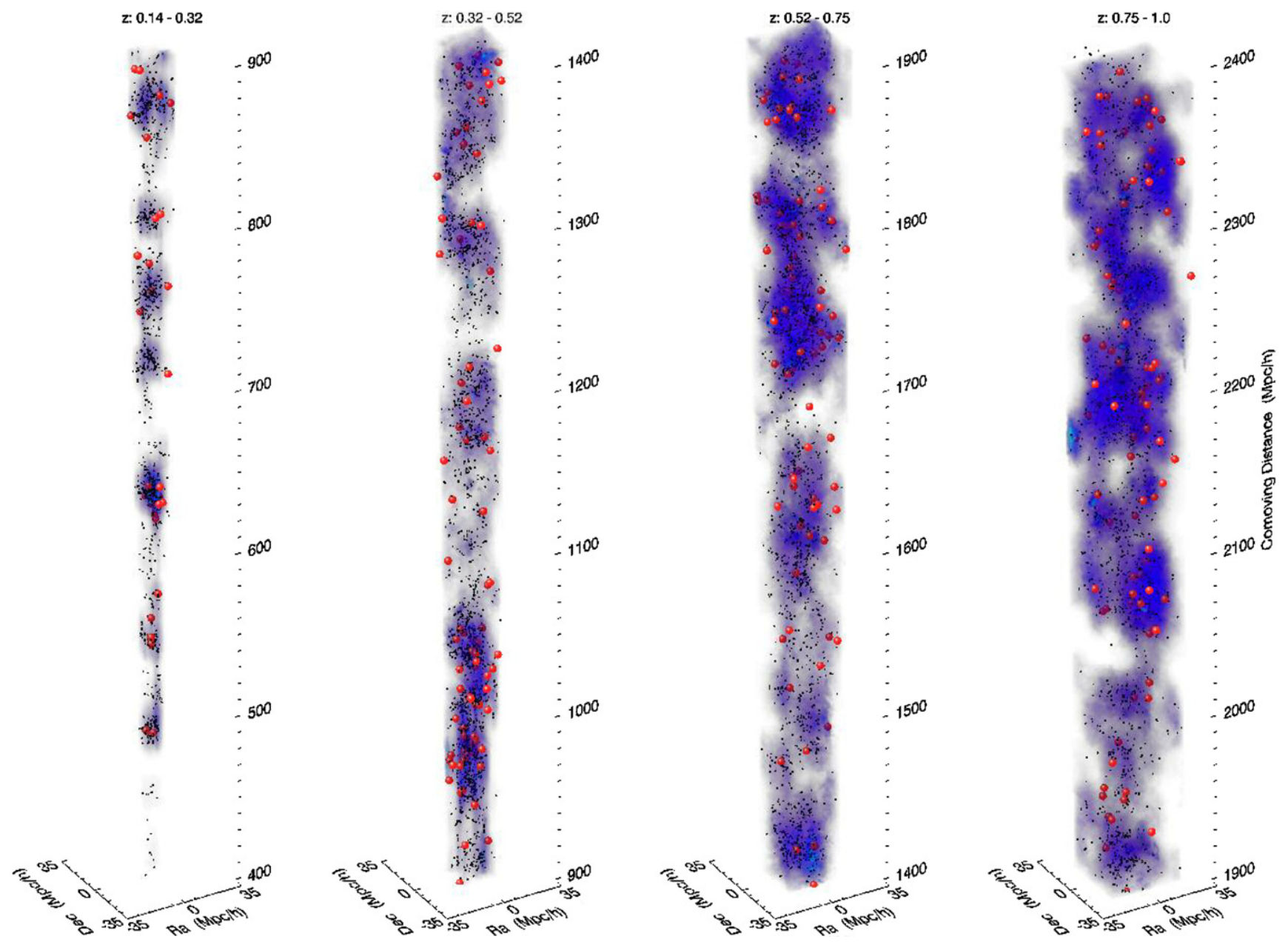

Figure 3. Three-dimensional distribution of AGN with respect to the zCOSMOS overdensity distribution. Each panel shows a different redshift interval as labeled. X-ray-selected AGN, identified by both the zCOSMOS and Magellan/IMACS (Trump et al. 2007) programs in the COSMOS field, are marked in red, while zCOSMOS galaxies are shown by the small black dots.

It is important to consider if any difference in spatial sampling exists between the AGN and randomly selected galaxies. Such an effect may be inherently induced by the VIMOS automated slit assignment software when designating sources as "compulsory," as done for a subset of the X-ray sources. We do find that the number of randomly observed galaxies in the vicinity of "compulsory" targets is lower than that of the random sample. This is slightly noticeable in Figure 1 where the surface density of AGNs is not as concentrated toward the center of the field as the galaxies. This effect is both small and compensated by the "ZADE" approach thus we do not expect any significant bias in our density estimates.

In Figure 3, we show the zCOSMOS density field in comoving coordinates with the location of galaxies and those hosting AGN as marked. For display purposes, the overdensity distribution, estimated on a grid $(\Delta \alpha=\Delta \delta=1 \operatorname{arcmin}, \Delta z=0.002)$ has been interpolated to a comoving scale of $1.4 \mathrm{Mpc}$. It is clearly evident that the COSMOS field encompasses a wide range of environments from voids to dense structures. The survey area $\left(\sim 2 \mathrm{deg}^{2} ; \sim 80\right.$ comoving Mpc at $\left.z \sim 1\right)$ is still narrow enough that sheets, filaments, and walls appear to cut across the field.

\section{4. zCOSMOS Galaxy Groups}

A galaxy group catalog (Knobel et al. 2009) has been generated using the 10k "bright" catalog to determine the role of environment in galaxy evolution (K. Kovač et al. 2009, in preparation; A. Iovino et al. 2009, in preparation). This approach is complementary to that based on the density field due to its ability to probe smaller physical scales. Two group finding algorithms (friends-of-friends, Voronoi-Delaunay Method) are employed to minimize effects such as group fragmentation, overmerging, spurious detections, or missed groups. Extensive testing on the aforementioned mock catalogs is done for optimization of the algorithms. For each group, its properties (velocity dispersion, dynamical mass, corrected richness) are determined with a level of uncertainty $\sim 20 \%$ due to the small number of observed group members $(\sim 2-12)$ reflective of the bright magnitude limit of the survey. A final catalog of 800 groups is constructed with at least two members using the friends-of-friends technique well tuned using the methods employed above. The completeness (i.e., detected group members relative to actual members) has been assessed to be $\sim 70 \%-90 \%$ (see Figure 8 of Knobel et al. 2009) and fairly constant with respect to the number of group members. The number of interlopers is estimated to be $\sim 20 \%$. The redshift distribution of the final group catalog is very similar to the overall redshift distribution of zCOSMOS galaxies with two prominent features at $z \sim 0.3$ and $z \sim 0.7$. The masses span an interval $\sim 10^{12}-10^{14} M_{\odot}$ (see Figures 13 and 15 of Knobel et al. 2009), a regime below that of local, massive $\left(\gtrsim 10^{14} M_{\odot}\right)$ clusters. 

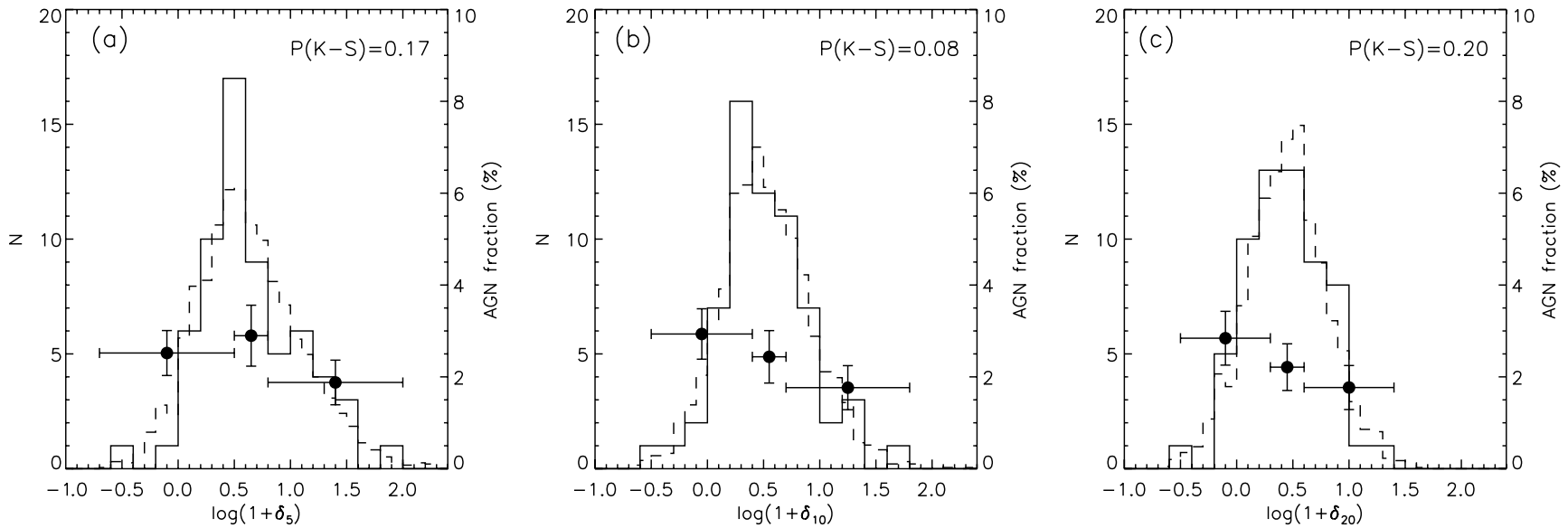

Figure 4. Overdensity distribution of a mass-selected sample of 2457 galaxies (Sample A: $\log M_{*}>10.4 ; 0.1<z<1.0$; dashed histogram) and those that host $\operatorname{AGN}\left(63 ; 42.48<\log L_{0.5}-10.0 \mathrm{keV}<43.7\right.$; solid histogram) using the 5th $(a), 10$ th, $(b)$ and 20 th $(c)$ nearest neighbors. In each panel, the underlying parent galaxy population has been scaled to match the number of AGNs. The results from a K-S test on the observed distributions are given in each panel. The data points are a measure of the fraction of galaxies hosting AGN with $1 \sigma$ errors and horizontal bars denoting the bin size.

\section{LARGE-SCALE ENVIRONMENTS OF AGN}

In Figure 4, we present the distribution of zCOSMOS galaxies and those hosting X-ray-selected AGN (Table 1; sample A) as a function of their environment (i.e., overdensity). The results are shown using the distance to the nth nearest neighbor (5th, 10th, and 20th) separately that probes a range of comoving scales $(\sim 1-4 \mathrm{Mpc})$. In all panels (Figures 4(a)-(c)), we generally see that AGNs reside in a broad range of environments (e.g., panel (a) $0 \lesssim \log 1+\delta \lesssim 1.5$ ) remarkably similar to the parent galaxy population of equivalent stellar mass ( $\left.\log M_{*}>10.4\right)$ as substantiated by the low probability (less than $20 \%$ in each case), based on a Kolmogorov-Smirnov (K-S) test, that we can reject the null hypothesis (i.e., two distributions are drawn from the same population). To avoid any possible luminosity-dependent systematics, we perform additional $\mathrm{K}-\mathrm{S}$ tests in fine redshift bins $(\Delta z=0.1)$ over the redshift range $0.3<z<0.8$; the probability $(P=0.25,0.49,0.54,0.39$, and 0.03$)$ that the two distributions (overdensities based on the 5 th nearest neighbor) are different conclusively supports this finding.

To further investigate if any environment influences are present, we measure the fraction of galaxies that host AGN as a function of overdensity. We follow the technique discussed in Section 3.1 of Lehmer et al. (2007) to determine the AGN fraction for our parent population of galaxies. This method properly accounts for the spatially varying sensitivity limits of the XMM observations of the COSMOS field (Figure 1). The necessity of this approach is demonstrated in Figure 1 of Silverman et al. (2009) that shows the limiting X-ray luminosity as a function of redshift for the entire galaxy sample and the measured X-ray luminosities of those galaxies harboring AGN. The sensitivity of the $X M M$ coverage is remarkably uniform as shown by the relatively narrow distribution of the X-ray limits at each redshift. To properly account for the luminosity-redshift relation, we determine the contribution of each AGN separately to the total fraction. The AGN fraction $(f$; see Equation (1) below) is determined by summing over the full sample of AGN $(N)$ with $N_{\text {gal, }}$ representing the number of galaxies in which we could have detected an AGN with X-ray luminosity $L_{\mathrm{X}}^{i}$. The sampling rate of the random galaxies $\left(S_{\mathrm{gal}}, 29.8 \%\right)$ and AGN (i.e., X-ray sources, $S_{x} ; 71.9 \%$ ) are incorporated since these differ due to the fact that $54 \%$ of the X-ray sources are designated as "compulsory targets" when designing masks for VIMOS. We estimate the associated $1 \sigma$ error (Equation 2) using binomial statistics where $N_{\mathrm{AGN}}^{\mathrm{eff}}$ is the number of AGNs that would be detected if all galaxies have the same limiting X-ray sensitivity and the sample of AGN was randomly selected. Here, we only consider AGN with $42.48<\log L_{0.5-10 \mathrm{kev}}<43.7$. The lower limit ensures that we have a statistically significant sample of parent galaxies $(\gtrsim 700)$ that could host each AGN while the upper limit restricts the sample to low-to-moderate luminosities thus securing the accuracy of their host-galaxy masses. We refer the reader to Silverman et al. (2008a) for further details and results employing this method:

$$
\begin{gathered}
f=\sum_{i=1}^{N} \frac{1 / S_{x}}{N_{\mathrm{gal}, \mathrm{i}} / S_{\mathrm{gal}}} \\
\sigma^{2}=N_{\mathrm{AGN}}^{\mathrm{eff}} \times\left(N_{\mathrm{gal}}-N_{\mathrm{AGN}}^{\mathrm{eff}}\right) / N_{\mathrm{gal}}^{3} .
\end{gathered}
$$

In Figures 4(a)-(c), we give the results of this exercise with the fraction of galaxies hosting AGN as measured in three bins of overdensity with widths set to have equal numbers of galaxies therein. In agreement with the previous analysis, we find that the AGN fraction is not strongly enhanced at any particular value of overdensity. The AGN fraction is low $(\sim 2 \%-4 \%)$ compared to similar studies (e.g., Kauffmann et al. 2004) and due to our selection in a narrow range of X-ray luminosity. We finally remark that an underlying dependence on environment for a subset of our sample is realized as demonstrated in the next section and possibly seen here by the slight trend in the AGN fraction (Figures 4(b) and (c)).

We further test whether an environmental influence is present for AGN of a specific X-ray luminosity or hardness ratio (HR). The HR is a means of easily characterizing the X-ray spectrum (i.e., level of absorption) by measuring the ratio of X-ray counts in the hard band $(\mathrm{H} ; 2.0-10 \mathrm{keV})$ relative to the soft band (S; $0.5-2.0 \mathrm{keV}): \mathrm{HR}=(\mathrm{H}-\mathrm{S}) /(\mathrm{H}+\mathrm{S})$. In Figure 5, we plot local overdensity versus $\mathrm{X}$-ray luminosity while highlighting hard $(\mathrm{HR}>-0.2)$ sources. We find that there is no correlation between galaxy environment and the intrinsic AGN emissivity or any absorbing material.

\subsection{High-Mass Galaxies}

We investigate whether there exists a relation between AGN activity and environment for galaxies of a specific stellar mass. 


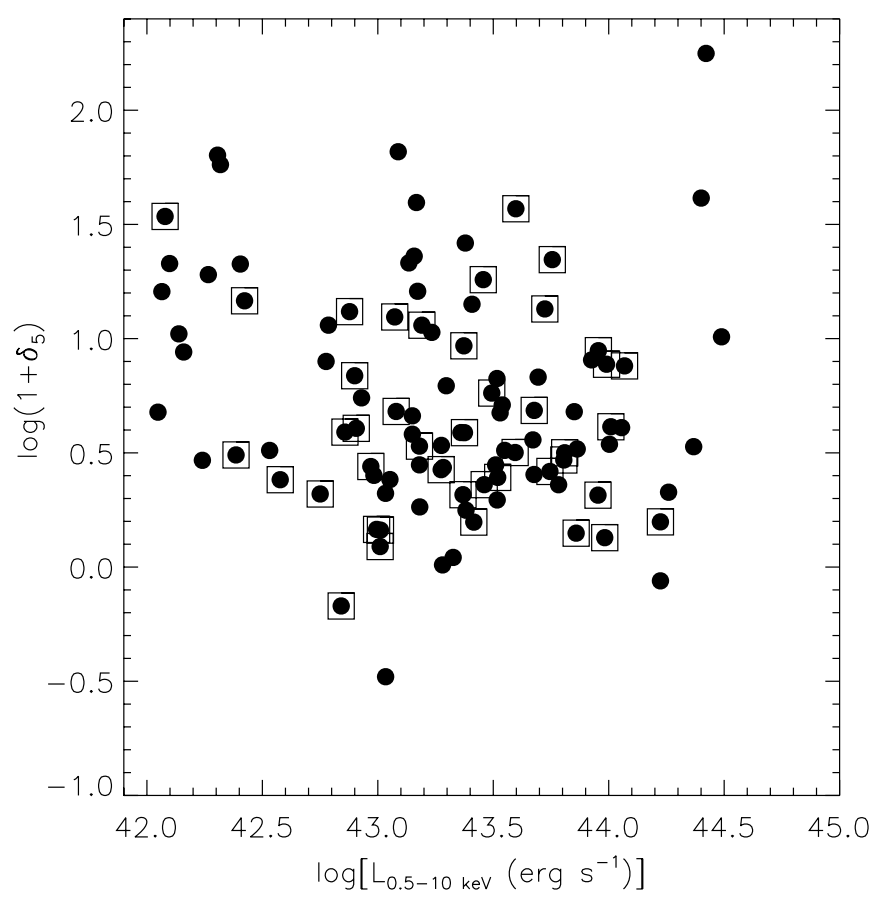

Figure 5. Overdensities using the 5th nearest neighbor for galaxies hosting AGN as a function of their X-ray luminosity. Absorbed AGNs $(\mathrm{HR}>-0.2)$ are further marked by an open square. There are no environmental influences depending on the intrinsic properties of AGN.
Our motivation is based on the mass-dependent environmental relationship seen in SDSS studies (Kauffmann et al. 2004). In contrast to our analysis in the previous section, we include more X-ray luminous AGN $\left(\log L_{0.5}-10 \mathrm{keV}>42.48\right)$ that have optical spectra dominated by their host galaxy as determined by visual inspection thus slightly improving our statistics for the highest mass galaxies. In Figure 6, we show plots equivalent to those presented in the previous section and split into two mass bins (panels (a)-(c): $10.4<\log M_{*}<11$; panels (d)-(f): $\left.\log M_{*}>11\right)$ with the statistics given in Table 1 (Samples B, C). For the lower-mass interval (Figures 6(a)-(c)), we find that the results are consistent with our previous findings and the AGN fraction is remarkably similar over the full range of overdensities. On the other hand, the overdensity distribution (Figures 6(d)-(f)) of galaxies hosting AGN is dissimilar to the underlying massive galaxy population due to the lack of AGNs in higher-density environments $\log (1+\delta>0.5)$. The difference in these distributions is significant at the 2.3-2.5 $\sigma$ level based on the probabilities $(0.99,0.98)$ from $\mathrm{K}-\mathrm{S}$ tests using either the 5 th or 10 th nearest neighbor. We note that these results are still apparent when restricting the analysis to lower-luminosity AGN although at a slightly reduced level of significance $(2 \sigma)$. Therefore, the fraction of galaxies hosting AGN is higher in underdense regions $(\log (1+\delta<0.5)$ since the offset between the distributions is substantially greater than typical errors in overdensity. These results may further explain the discrepancy between the environmental studies based on the SDSS (Miller et al. 2003; Kauffmann et al. 2004). We remark that our current sample of massive galaxies $\left(\log M_{*}>11\right)$ in zCOSMOS with
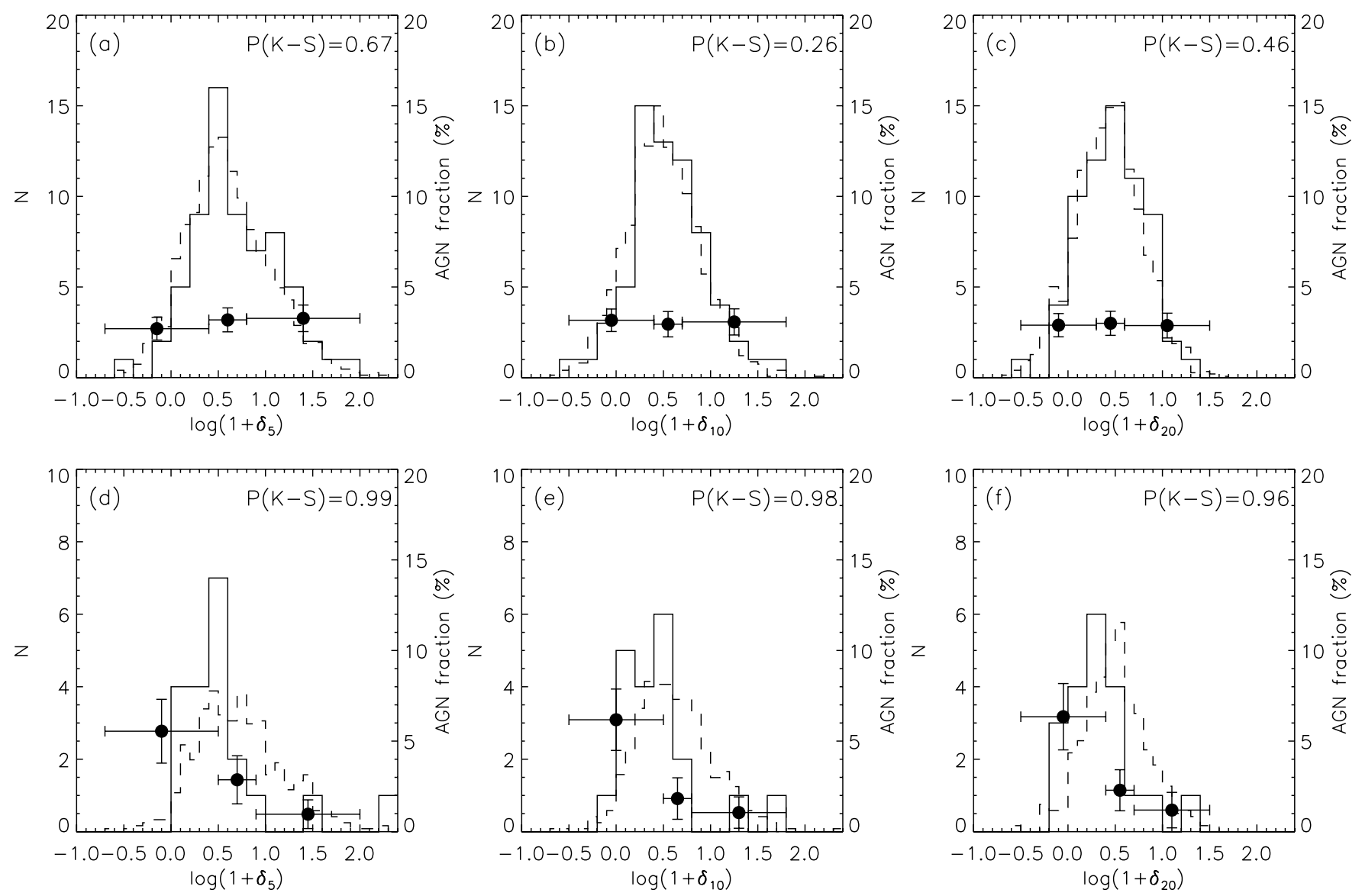

Figure 6. Overdensity distribution of galaxies $(0.1<z<1.0)$ and those hosting AGN as a function of stellar mass: (Sample B: panels $(a)-(c)) 10.4<\log M_{*}<11.0$, (Sample C: panels $(d)-(f)) \log M_{*}>11.0$. The histograms and data points are the same as given in Figure 4 . The results from a K-S test are given in each panel. 

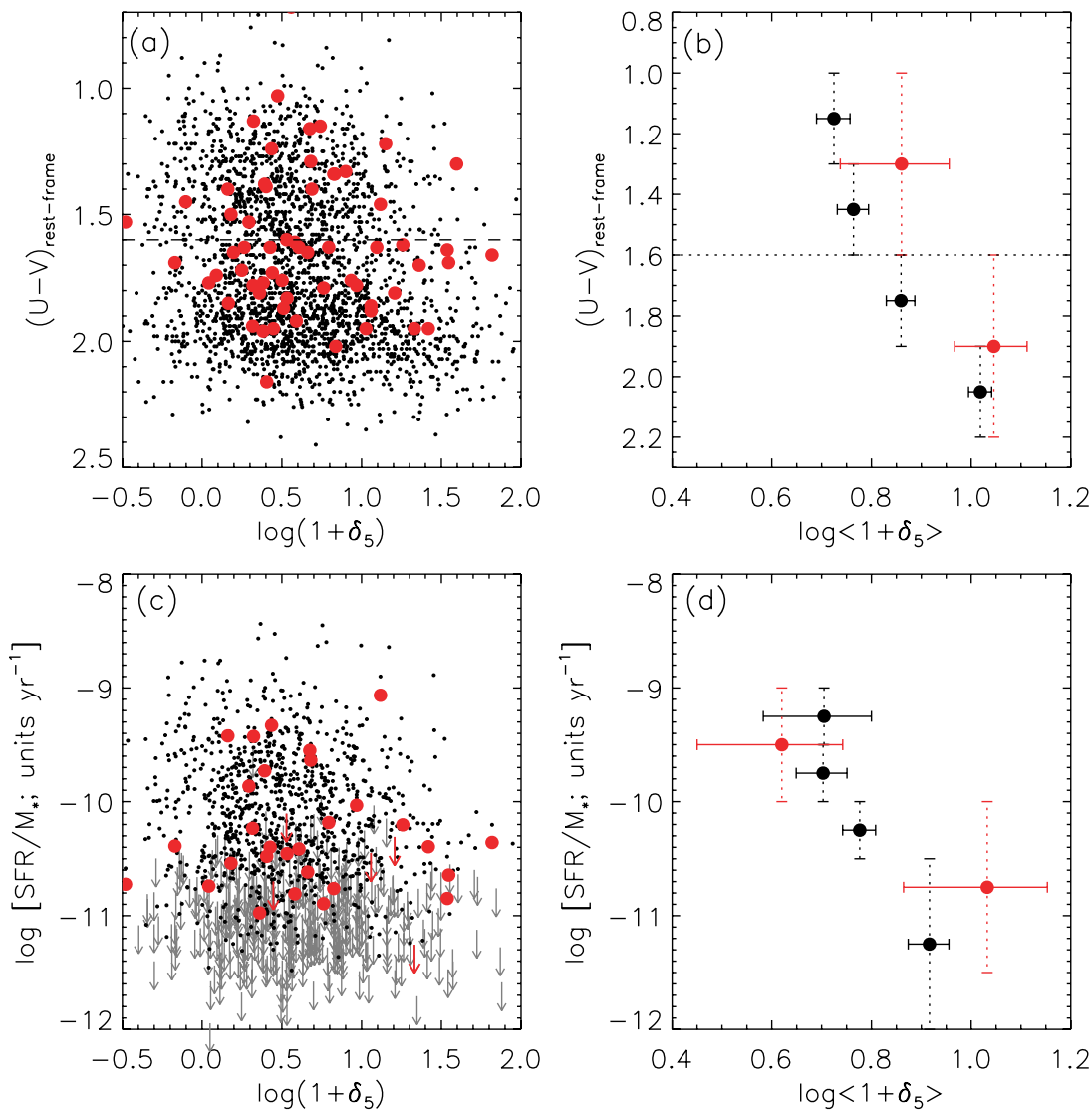

Figure 7. (a) Color-density relation of galaxies (small black circles; Table 1; Sample D) and those with AGN (large red circles). (b) Mean overdensity and associated error for color bins marked by the vertical dashed lines. The symbol colors are the same as in panel $(a)$. The AGN sample is split into two color bins that essentially represent "blue cloud" and red sequence galaxies with a fixed color division $(U-V=1.6)$. (c) sSFR-density relation (arrows are upper limits) with mean values given in panel $(d)$. We find that the host galaxies appear to exhibit a color/sSFR-density relation similar to the underlying galaxy population although improved statistics are required to firmly justify such a conclusion.

AGN is small (20) and will improve with the full zCOSMOS 20k catalog and deeper Chandra observations.

\subsection{Dependence on Stellar Content of AGN Hosts}

The result that AGN prefers to reside in lower-density environments for those having massive host galaxies is reminiscent of the well-known color-density relation for galaxies in general. To explore this aspect further, we plot the distribution of rest-frame color $U-V$ versus overdensity in Figure 7. For the following analysis, we decrease our mass $\operatorname{limit} \log M_{*}>10.2$ in order to improve upon the numbers of AGN hosts with blue colors thus requiring us to reduce the maximum redshift to $z=0.8$ to not induce a color bias (Table 1; Sample D). In panel (a), galaxies exhibit an environmental influence with red galaxies $(U-V>1.6)$ having a prominent extension of their overdensity distribution toward higher values $\log \left(1+\delta_{5} \gtrsim 1.0\right)$. The well-known trend of increasing density for redder galaxies (i.e. color-density relation) is clearly seen in panel (b) where we measure the mean overdensity for galaxies in bins of rest-frame color (black points).

We test whether galaxies hosting AGN exhibit a similar color-density relation as described above. AGN hosts are marked appropriately in Figure 7. From their mean overdensities (panel (b)), AGNs appear to follow a similar relation to the nonactive galaxies although the errors are substantial with a significance of $1.7 \sigma$ in the difference (see Georgakakis et al. 2007, for an equivalent analysis and conclusion). In support of the color-density relation for AGN hosts, we investigate the connection between ongoing star formation and environment. In Figure 7(c), we plot the mass-weighted "specific" SFR (sSFR), a quantity based on [O II] 33727 luminosity, versus galaxy overdensity. The mean overdensity in bins of sSFR is calculated and shown by the large black dots in panel (d). We find a similar result and uncertainty as above with the environment being related to the stellar content of the hosts of AGN. It is worth highlighting that a clear dichotomy is present between the importance of both mass and environment in determining the likelihood of a galaxy's properties (see Cucciati et al. 2009, in preparation for further analysis based on zCOSMOS galaxies).

Coupled with the results of our companion study (Silverman et al. 2009), AGN prefers to reside in galaxies undergoing star formation irrespective of their environment. We illustrate this conclusion in Figure 8 where the fraction of galaxies hosting AGN is shown as a function of rest-frame color $U-V$ and overdensity. Galaxies bluer $(U-V \lesssim 1.6)$ than those that have stopped forming stars (i.e., along the red sequence) have higher levels of AGN activity. We further find here that this result holds for AGN hosts residing in either lower- or higher-density environments.

By looking in detail at the most massive galaxies $\left(\log M_{*}>\right.$ 11; Figure 9), we can further understand the relation of AGNs to their environment and the presence of star formation. The locus of AGNs in the color-density plane (panel (a)) is offset from that of massive galaxies due to their bluer colors and lower 


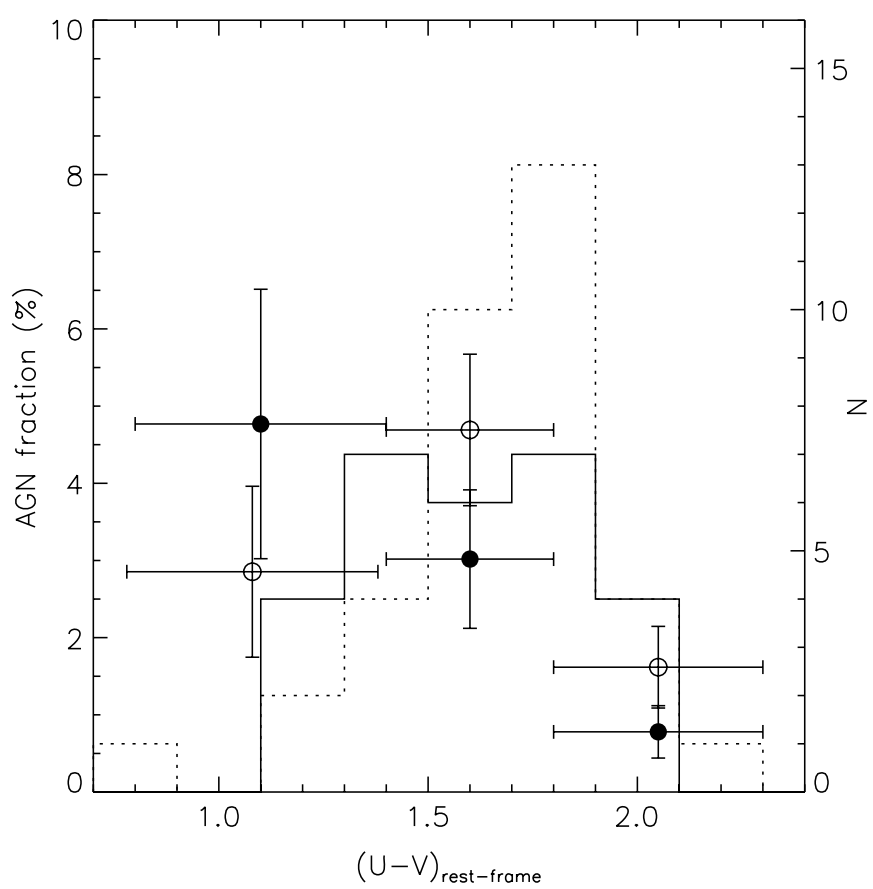

Figure 8. Fraction of galaxies hosting AGN as a function of rest-frame color $U-V$ and environment. Data points are given for environments above (filled circle) and below (open circle) $\log \left(1+\delta_{5}\right)=0.6$. The color distribution of AGNs in these two environmental categories is also shown (solid histogram: high-density, dashed histogram: lower density).

overdensities. Clearly, the distinction between the overdensity distribution of AGN hosts and their parent population is due to the fact that AGNs do not reside in red galaxies (panel (b)) that tend to live in denser environments but rather require fuel for accretion that tends to drive subsequent star formation. Therefore, the environments of AGNs hosts are similar to starforming galaxies $(c)$. We interpret these results as evidence that massive galaxies are more likely to have accreting SMBHs if not subjected to harsher environments that can effectively expel gas, due to processes related to galaxy interactions, thus impacting star formation and subsequent AGN activity.
Table 2

Sample Statistics-group Analysis

\begin{tabular}{lccc}
\hline \hline Environment & $\begin{array}{c}\text { Galaxies } \\
M_{*}>10^{10.4}\end{array}$ & AGN $^{\mathrm{a}}$ & AGN Fraction (\%) \\
\hline Field & 1675 & 63 & $3.28 \pm 0.43$ \\
Group & 828 & 27 & $2.59 \pm 0.55$ \\
Group $\left(R^{\mathrm{b}} \geqslant 1\right)$ & 614 & 21 & $2.89 \pm 0.68$ \\
$\left(R^{\mathrm{b}} \geqslant 2\right)$ & 491 & 19 & $3.17 \pm 0.79$ \\
$\left(R^{\mathrm{b}} \geqslant 4\right)$ & 237 & 10 & $3.00 \pm 1.11$ \\
\hline
\end{tabular}

\section{Notes.}

${ }^{a} \log M_{*}>10.4 ; \log L_{0.5-10 \mathrm{keV}}>42.48$

b Effective group richness.

\section{AGN CONTENT OF OPTICALLY SELECTED GROUPS}

The presence of AGN within well-defined structures such as groups (Georgakakis et al. 2008), clusters (Martini et al. 2007; Kocevski et al. 2009; Lehmer et al. 2009), or large-scale sheets/ filaments (Gilli et al. 2003; Silverman et al. 2008a) as compared to the field offer an alternative perspective on the environmental impact on AGN activity. To do so, we utilize the catalog of 800 galaxy groups (Knobel et al. 2009) as described in Section 2.4. As done in previous analyses, we restrict the sample to galaxies with stellar masses above $2.5 \times 10^{10} M_{\odot}$ thus resulting in a sample of 2444 galaxies with a designation as either a "field" or "group" galaxy. In Table 2, we list the numbers of galaxies in each subclass, those that host AGNs $\left(\log L_{0.5-10 \mathrm{keV}}>42.48\right)$ and the derived AGN fraction as detailed below.

We simply measure the fraction of galaxies hosting AGN for the "group" and "field" populations separately to test whether any environmental dependence exists. Over the full redshift range $(0.1<z<1.05)$, we measure the fraction to be $3.28 \% \pm 0.43 \%$ for field galaxies. For galaxies in groups, we find an AGN fraction of $2.59 \% \pm 0.55 \%$, based on two or more spectroscopically identified group members, slightly less than that in the field although not statistically significant. To account for redshift-dependent effects and spatial sampling in the group catalog, we further isolate groups having an effective (i.e., corrected) richness $\left(N_{\text {eff }}\right)$ defined as the number of galaxies above a given magnitude threshold (see Section 4.2 of Knobel
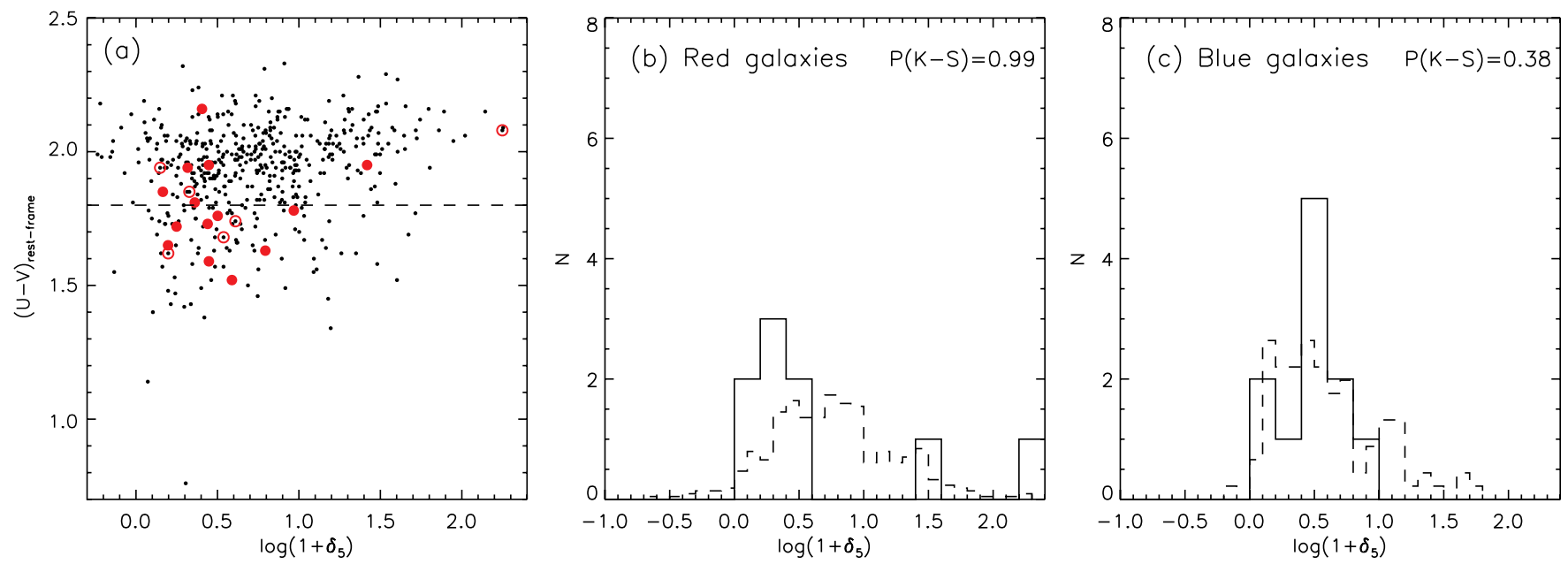

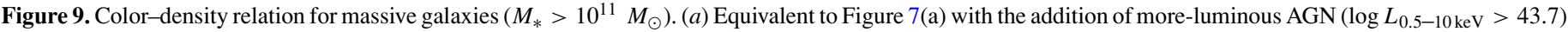

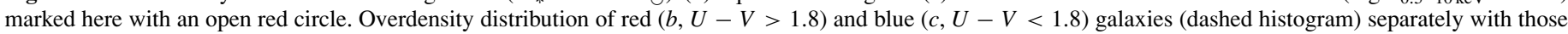
hosting AGN shown by the solid histogram. The probabilities based on $\mathrm{K}-\mathrm{S}$ tests are shown in the respective panel.

(A color version of this figure is available in the online journal.) 

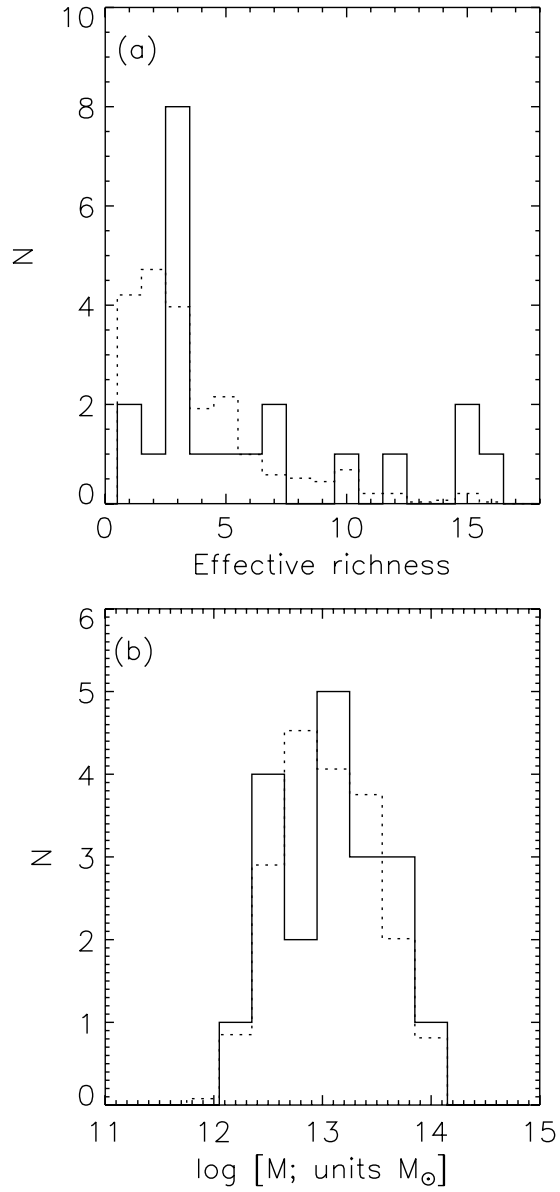

Figure 10. Distribution of group properties (a) effective richness; (b) mass) of galaxies hosting AGN (solid histogram) having $\log M_{*}>10.4$. For comparison, the distribution of all galaxies of similar masses has been renormalized and shown by the dashed histogram.

et al. 2009, for details) that can be detected over the full redshift range considered here. This limit is based on the absolute magnitude in the $b$-band $\left(M_{b, \lim }<20.5-z\right)$ and includes a redshift term to account for the luminosity evolution of galaxies. In Figure 10(a), we show the distribution of effective richness for all galaxies $\left(\log M_{*}>10.4\right)$ residing in groups that illustrate the scale of the zCOSMOS groups. In addition, the distribution of group mass (Figure 10(b)) is given. Briefly, the group mass is the mean mass of halos in the mock catalog that are associated with groups of a specific effective richness $\left(N_{\text {eff }}\right)$. A redshift dependence is inherent given that effective richness is based on an observed quantity (i.e., apparent magnitude). Full details regarding these derived properties can be found in Knobel et al. (2009). Considering a selection on effective richness, we find a similar fraction of galaxies hosting AGN as compared to the field with no obvious dependence on the effective richness (see Table 2) of the group. Furthermore, there is no significant difference in the X-ray luminosity or host-galaxy color (restframe $U-V$ ) distributions of AGNs in or out of groups (see Georgakakis et al. 2008, for similar analyses). These results are in agreement with our previous findings based on the density field that AGNs in galaxies with $M_{*}>10^{10.4} M_{\odot}$ do not show a strong dependence on environment.

To determine if any signs of evolution exist, we plot the fraction of group galaxies hosting $\mathrm{AGN}$ in two redshift bins (Figure 11). Here, we select groups having $N_{\text {eff }} \geqslant 2$ as described above. The AGN fraction is remarkably similar between for

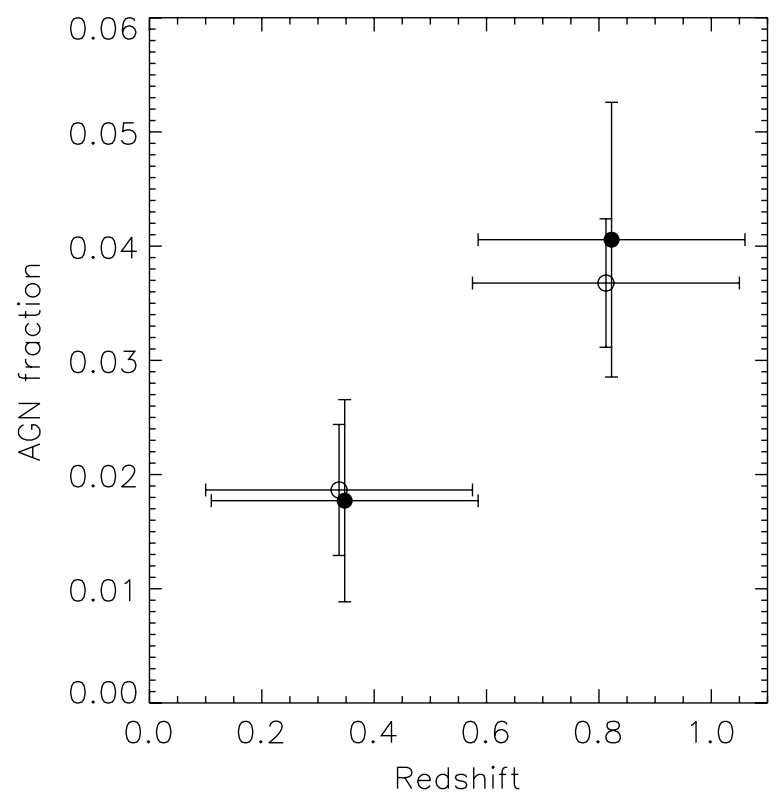

Figure 11. Fraction of galaxies ( $\left.\log M_{*}>10.4\right)$ hosting AGN split by those in galaxy groups ( $N_{\mathrm{eff}}>2$, filled circle) and those in the field (open circle). Errors are $1 \sigma$ based on binomial statistics.

galaxies in groups and in the field for both redshift intervals. The increase in the AGN fraction in the higher redshift bin for both the field and groups, is most likely due to the overall positive luminosity evolution of the AGN population (e.g., Barger et al. 2005; Silverman et al. 2008b). Therefore, we do not find an enhancement in the AGN content of groups with redshift as strong as reported for clusters (Eastman et al. 2007) above the level expected by the AGN luminosity function. As previously mentioned, we will improve upon the current sample, as justified by the substantial errors, in a future investigation by using the zCOSMOS 20k catalog and deeper Chandra observations (Elvis et al. 2009) that provide additional AGNs of moderate luminosity ( $42 \lesssim L_{X} \lesssim 43$ ) over the redshift range $0.5-1.0$. A larger sample will enable us to isolate high-mass galaxies $\left(M_{*}>10^{11} M_{\odot}\right)$ as done with the density field.

\section{CONCLUSION AND SUMMARY}

We have assessed whether the environment plays any role in regulating mass accretion onto SMBHs, on scales of a few megaparsecs, using 7234 galaxies $\left(i_{\mathrm{ACS}}<22.5\right.$; $\left.0.1<z<1.0\right)$ from the zCOSMOS survey ("bright" program). To do so, a parent sample of 2457 galaxies is carefully constructed based on stellar mass $\left(\log M_{*}>10.4\right)$ with no obvious color $(U-V)$ bias. Galaxies undergoing substantial accretion onto a SMBH are identified by the presence of an AGN with Xray emission detected by XMM-Newton; we find 147 AGN with 101 residing in host galaxies having $\log M_{*}>10.4$. With two characterizations of the local environment, we measure the fraction of galaxies as a function of their galaxy overdensity based on the zCOSMOS density field (Kovač et al. 2009) and presence in optically selected galaxy groups (Knobel et al. 2009).

Based on the zCOSMOS density field, we find that the AGNs, with exception of those residing in the most massive host galaxies $\left(\log M_{*}>11\right)$, span a broad range of environments (see Georgakakis et al. 2007 and Montero-Dorta et al. 2009, for similar conclusions), from the field to massive groups, equivalent to galaxies of similar stellar mass. Our 
findings do appear to be consistent with low redshift studies based on narrow-line AGN from the SDSS (Miller et al. 2003; Kauffmann et al. 2004; Constantin et al. 2008) that taken together demonstrate that the environments depend on the stellar mass of their host galaxy. The lack of an environmental dependence on AGN activity over the lower-mass regime $\left(10.4<\log M_{*}<11\right)$ in zCOSMOS is compatible with the results of Miller et al. (2003) since their magnitude-limited sample, dominated by low-luminosity AGN, most likely includes many lower-mass galaxies. An environmental dependence with AGN activity less common in higher-density regions emerges for "strong AGN" residing in galaxies of higher mass (Kauffmann et al. 2004; Constantin et al. 2008) and is also evident in our study based on zCOSMOS galaxies with $\log M_{*}>11$. We contend that the discrepant findings based on SDSS studies at low redshift may be due to the differences in the underlying mass distribution of galaxies rather than the differences of the AGN selection.

We find that the presence of young stars in the host galaxies of AGN (Kauffmann et al. 2003; Jahnke et al. 2004; Silverman et al. 2009) is also indicative of their environments. Galaxies hosting AGN in zCOSMOS follow a similar color-density relation to nonactive galaxies (Cucciati et al. 2009, in preparation) with blue hosts $(U-V<1.6)$ having a lower mean overdensity than red galaxies. An equivalent relation based on SFR or that weighted by stellar mass (specific) supports this assertion. As fully discussed in Cucciati et al. (2009, in preparation), the sSFR is seen to be more strongly dependent on environment than SFR due to the underlying mass dependence discussed above. The majority of massive $\left(\log M_{*}>11\right)$ AGN hosts having colors bluer than the underlying galaxy population agrees with their residence in lower-density environments. Therefore, we conclude that the incidence of AGN activity as a function of environment depends on the properties of its host galaxy namely its mass content both in the form of stars and gas in the interstellar medium.

Furthermore, we carry out a complementary analysis of the environments of AGN by utilizing the optically selected catalog of galaxy groups (Knobel et al. 2009) in zCOSMOS. Such groups have halo masses $\sim 10^{12}-10^{14} M_{\odot}$ similar to those that host the more-luminous quasars (e.g., Porciani et al. 2004; Pasquali et al. 2008; Bonoli et al. 2008) and less massive than clusters at low redshift. We measure the incidence of AGN activity in galaxies in groups compared to that in the "field." This method effectively enables us to probe smaller physical scales $(\lesssim 1 \mathrm{Mpc})$ not possible with the density field due to the spatial resolution of $\pm 1000 \mathrm{~km} \mathrm{~s}^{-1}$ along the line of sight. An excess signal around quasars, based on either near-neighbor counts (Serber et al. 2006) or the quasar correlation function (Hennawi et al. 2006), highlights the importance of probing scales $\sim 100 \mathrm{kpc}$. Although, the environments of $31 \mathrm{X}$-rayselected AGN (Waskett et al. 2005) with $0.4<z<0.6$ from the Canada-France-Redshift Survey fields are indistinguishable from a well-matched control sample of galaxies on scales around 30-500 kpc.

We find in zCOSMOS that AGNs are essentially equally likely to reside in or out of a galaxy group irrespective of its properties (i.e., effective richness). The fraction of group galaxies ( $\left.\log M_{*}>10.4\right)$ hosting an AGN is similar to "field" galaxies $(\sim 3 \%)$. This is in agreement with both our results based on the density field for galaxies spanning the full mass range $\log M_{*}>10.4$, and those of Georgakakis et al. (2008) which consider the host-galaxy properties. We note that the current AGN sample in zCOSMOS residing in galaxy groups is too small to investigate if an environmental effect for the most massive galaxies exists as exercised using the density field. Our lack of a strong environmental effect is also consistent with the incidence of X-ray-selected AGN in galaxy clusters (Martini et al. 2007) as compared to the field. Also, an increase in the AGN fraction in the higher of two redshift bins is significantly smaller than the enhancement reported for galaxy clusters (Eastman et al. 2007) and in our case most likely due to the luminosity evolution of the AGN population since both the group and field samples show an increase of equal magnitude.

We conclude that internal processes (Hopkins \& Hernquist 2006) play an important role in the growth of SMBHs during an AGN phase of moderate luminosity $\left(L_{X} \sim 10^{43} \mathrm{erg} \mathrm{s}^{-1}\right.$; "Seyfert mode") that accounts for the bulk of the Cosmic X-ray Background (e.g., Gilli et al. 2007). Major mergers of galaxies, possibly relevant for the more-luminous quasar phenomenon, may not be the primary mechanism for fuelling these AGN due to the lack of any enhancement of activity in specific environments likely to be conducive for merging (i.e., galaxy overdensities comparable to the small group scale). In the opposite sense, we do find that for the most massive galaxies $\left(\log M_{*}>11\right)$ the environment plays a role possibly through various physical processes (e.g., tidal stripping, harassment) in higher-density environments that concurrently shuts down star formation and accretion onto supermassive black holes. This highlights the requirements for a galaxy to host an accreting SMBH, as observed by their X-rays, a massive bulge (e.g., Sanchez et al. 2004; Grogin et al. 2005; Silverman et al. 2008a; Gabor et al. 2009), and a sufficient fuel supply as indicated by the young stars usually present in AGN host galaxies (Kauffmann et al. 2003; Silverman et al. 2009).

We thank the referee and Paul Martini for constructive comments that significantly improved the paper. This work is fully based on observations undertaken at the European Southern Observatory (ESO) Very Large Telescope (VLT) under the Large Program 175.A-0839 (P.I.: Simon Lilly).

Facilities: XMM, VLT:Melipal (VIMOS)

\section{REFERENCES}

Baldry, I. K., Balogh, K., Bower, R. G., Glazebrrok, K., Nichol., R. C., Bamford, S. P., \& Budavari., T. 2006, MNRAS, 373, 469

Barger, A., Cowie, L. L., Mushotzky, R. F., Yang, Y., Wang, W.-H., Steffen, A. T., \& Capak, P. 2005, AJ, 129, 578

Barnes, J., \& Hernquist, L. 1996, ApJ, 471, 115

Best, P. N., von der Linden, A., Kauffmann, G., Heckman, T., \& Kaise, C. R. 2007, MNRAS, 379, 894

Bonoli, S., Marulli, F., Springel, V., White, S. D. M., Branchini, E., \& Moscardini, L. 2008, arXiv:0812.0003

Bower, R. G., Benson, A. J., Malbon, R., Helly, J. C., Frenk, C. S., Baugh, C. M., Cole, S., \& Lacey, C. G. 2006, MNRAS, 370, 645

Brusa, M., et al. 2007, ApJS, 172, 353

Bruzual, G., \& Charlot, S. 2003, MNRAS, 344, 1000

Calzetti, D., Armus, L., Bohlin, R. C., Kinney, A. L., Koornneef, J., \& StorchiBergmann, T. 2000, ApJ, 533, 682

Capak, P., et al. 2007, ApJS, 2007, 172, 99

Cappelluti, N., et al. 2007, ApJS, 172, 341

Cappelluti, N., et al. 2009, A\&A, in press (arXiv:0901.2347)

Coil, A. L., Hennawi, J. F., Newman, J. A., Cooper, M. C., \& Davis, M., ApJ, 654,115

Constantin, A., Hoyle, F., \& Vogeley, M. S. 2008, ApJ, 673, 715

Cooper, M. C., et al. 2006, MNRAS, 370, 198

Dressler, A., Thompson, I. B., \& Shectman, S. A. 1985, ApJ,, 288, 481 
Eastman, J., Martini, P., Sivakoff, G., Kelson, D. D., Mulchaey, J. S., \& Tran, K.-V. 2007, ApJ, 664, 9

Elvis, M., et al. 2009, ApJS, in press (arXiv:0903.2062)

Gabor, J., et al. 2009, ApJ, 691, 705

Gilmour, R., Gray, M. E., Almaini, O., Best, P., Wolf, P., Meisenheimer, K. Papovich, C., \& Bell, E. 2007, MNRAS, 380, 1467

Georgakakis, A., Gerke, B. F., Nandra, P., Laird, E. S., Coil, A. L., Cooper, M. C., \& Newman, J. A. 2008, MNRAS, 391, 183

Georgakakis, A., et al. 2007, ApJ, 660, 15

Gilli, R., Comastri, A., \& Hasinger, G. 2007, A\&A, 463, 79

Gilli, R., Zamorani, G., \& Miyaji, T. 2009, A\&A, 494, 33

Gilli, R., et al. 2003, ApJ, 592, 721

Granato, G. L., De Zotti, G., Silva, L., Bressan, A., \& Danese, L. 2004, ApJ, 600,580

Grogin, N., et al. 2005, MNRAS, 627, 97

Hasinger, G., et al. 2007, 172, 29

Hill, G. J., \& Lilly, S. J. 1991, ApJ, 367,

Hennawi, J. F., et al. 2006, ApJ, 131, 1

Hopkins, P. F., \& Hernquist, L. 2006, ApJS, 166, 1

Hopkins, P. F., Hernquist, L., Cox, T. J., \& Kereš, D. 2008, ApJ, 175, 356

Hopkins, P. F., Lidz, A., Hernquist, L., Coil, A., Myers, A. D., Cox, T. J., \& Spergel, D. N. 2007, ApJ, 662, 110

Jahnke, K., Kuhlbrodt, B., \& Wisotzki, L. 2004, MNRAS, 352, 399

Kauffmann, G., White, S., Heckman, T., Ménard, B., Brichmann, J, Charlot, S., Tremonti, C., \& Brinkmann, J. 2004, MNRAS, 353, 713

Kauffmann, G., et al. 2003, MNRAS, 346, 1055

Kitzbichler, M. G., \& White, S. D. M. 2007, MNRAS, 376, 2

Kocevski, D. D., Lubin, L. M., \& Gal, R. 2009, ApJ, 690, 295

Kovač, K., et al. 2009, ApJ, submitted (arXiv:0903.3409)

Knobel, C., et al. 2009, ApJ, in press (arXiv:0903.3411)

Lamareille, F., et al. 2008, A\&A (arXiv:0811.2053)

Lehmer, B. D., et al. 2007, ApJ, 657, 681

Lehmer, B. D., et al. 2009, ApJ, 691, 687

Li, C., Kauffmann, G., Heckman, T. M., Jing, Y. P., \& White, S. D. M. 2008, MNRAS, 385, 1903
Lilly, S. J., et al. 2007, ApJS, 172, 70

Maier, C., et al. 2009, ApJ, in press (arXiv:0901.0550)

Mandelbaum, R., Li, C., Kauffmann, G., \& White, S. D. M. 2008, arXiv:0806.4089

Martini, P., Mulchaey, J. S., \& Kelson, D. D. 2007, ApJ, 664, 761

Mihos, C., \& Hernquist, L. 1996, ApJ, 464, 641

Miller, C. J., Nichol, R. C., Gómez, P. L., Hopkins, A. M., \& Bernardi, M. 2003, ApJ, 597, 142

Montero-Dorta, A. D., et al. 2009, MNRAS, 392, 125

Moustakas, J., Kennicutt, R. C., \& Tremonti, C. A. 2006,, 642, 775

Padmanabhan, N., White, M., Norberg, P., \& Porciani, C 2008, arXiv:0802.2105

Porciani, C., Magliocchetti, M., \& Norberg, P. 2004, MNRAS, 355, 1010

Pasquali, A., van den Bosch, F. C., \& Mo, H. J. 2009, MNRAS, 394, 38

Pasquali, A., van den Bosch, F. C., Mo, H. J., Yang, X., \& Somerville, R. 2008, arXiv:0807.4178

Sanchez, S. F., et al. 2004, ApJ, 614, 586

Sanders, D., et al. 2007, ApJS, 172, 86

Schawinski, K., et al. 2007, MNRAS, 382, 1415

Scodeggio, M., et al. 2005, PASP, 117, 1284

Scoville, N., et al. 2007, ApJS, 172, 1

Serber, W., Bahcall, N., Ménard, B., \& Richards, G. 2006, ApJ, 643, 68

Springel, V., et al. 2005, Nature, 435, 629

Silverman, J. D., et al. 2005, ApJ, 618, 123

Silverman, J. D., et al. 2008a, ApJ, 675, 1025

Silverman, J. D., et al. 2008b, ApJ, 679, 118

Silverman, J. D., et al. 2009, ApJ, in press (arXiv:0810.3653)

Somerville, R. S., Hopkins, P. F., Cox, T. J., Robertson, B. E., \& Hernquist, L. 2008, MNRAS, 391, 481

Tremonti, C. A., et al. 2004, ApJ, 613, 898

Trump, J., et al. 2007, ApJS, 172, 383

van der Wel, A. 2008, ApJ, 675, 13

Waskett, T. J., Eales, S. A., Gear, W. K., McCracken, H. J., \& Lilly, S. 2005, MNRAS, 363, 801 